\title{
2020 closure, reviewers' gratitude, and improved review process transparency
}

\author{
Yoram Reich ${ }^{1}$
}

Published online: 25 January 2021

(c) The Author(s), under exclusive licence to Springer-Verlag London Ltd. part of Springer Nature 2021

How is it possible to write a closure to a year, in the midst of a pandemic that still dominates our life? We start with the dry facts.

Over the last year, the number of submissions to Research in Engineering Design dropped by several percent. We published 23 papers, $15 \%$ less than the year before. We definitely need to attract more quality submissions and make sure we reduce the review time to do it. One way that will help us monitor the review process better and present it transparently to authors is to incorporate new notifications to editors and authors about the progress of reviewing a paper including reviewers' assignments, reviewers' submission of reviews, etc.

None of this would have been possible without the extended community supporting the journal: the authors that trust the results of their research with the journal team, the area editors and the editorial board members, and the many dedicated reviewers. In a year challenged by a global pandemic, where many of us had to drastically change the way we teach and work, all of us were affected: those that conduct research and report the results of their research, those that manage the review of papers, those who review them, as well as the publisher's team. None of the tasks we did this year was the same as in the past.

I wish all of us good health and safe journey through this time and would like, especially, to extend my deep gratitude to the numerous reviewers who contributed their efforts to maintain and improve the quality of the journal. The reviewers, outside the editorial board, who completed a review this year, are:
Ivo Adan

Saeema Ahmed-Kristensen

Janet Allen

Jan Aurich

Niccolò Becattini

Amar Kumar Behera

Eric Blanco

Matt Bohm

Jean Francois Boujut

Tyson Browning

Anaelle Camarda

Felician Campean

Raul Carlsson

Denis Cavallucci

Peter Childs

Vicente Chulvi

Edoardo Filippo Colombo
Steve Culley

Oliver de Weck

Dov Dori

Chris Earl

Boris Eisenbart

Avner Engel

Scott Ferguson

Katherine $\mathrm{Fu}$

Shuichi Fukuda

Jose Jorge Garcia Agis

Georgi Georgiev

Kilian Gericke

Stefan Goetz

Paul Grogan

Peihua Gu

Claus Hansen

Pauline Herder
Katja Holtta-Otto

Christopher Hoyle

Ola Isaksson

Santosh Jagtap

Yan Jin

Nitin Joglekar

Shraddha Joshi

Udo Kannengiesser

Konstantinos Katsikopoulos

Sonal Keshwani

Timoleon Kipouros

Edwin Koh

Ehud Kroll

Torben Lenau

Simon Li

Julie Linsey

Yan Liu

Yoram Reich

yoramr@tauex.tau.ac.il

1 Tel Aviv University, Tel Aviv, Israel 
Ying-Chieh Liu

Junfeng Ma

Ivan Mactaggart

Tomi Mannisto

Tucker J. Marion

Franck Marle

Tomislav Martinec

Daniel McAdams

John McCardle

Chris McComb

Jay McCormack

Christophe Merlo

Greg Mocko

Yaniv Mordecai

Beshoy Morkos

Dimitris Mourtzis

Yukari Nagai

Alessia Napoleone

Monia Niero

Masaki Ogura

Maicon Oliveira

\author{
Kevin Otto \\ Massimo Panarotto \\ Vijitashwa Pandey \\ Pedro Parraguez Ruiz \\ Marina Proske \\ Karthik Ramani \\ BSC Ranjan \\ Adam Ross \\ Federico Rotini \\ Anna Rylander Eklund \\ Filippo Salustri \\ Prabir Sarkar \\ Carolyn Seepersad \\ Colleen Seifert \\ Yakov Shabi \\ Avi Shaked \\ Sanjiv Sharma \\ Murtuza Shergadwala \\ Miri Sitton \\ Chris Snider \\ Durward Sobek, II
}

Julia Sokol

Svenja Sommer

Martin Steinert

Manfred Tacker

Maxime Thomas

Christine Toh

Jakob Trauer

Cameron Turner

AMM Sharif Ullah

David Ullman

Noe Vargas Hernandez

Srinivasan Venkataraman

Pieter Vermaas

Vimal Viswanathan

Zheng Wang

Christian Weber

Kate Whitefoot

Jenny Whyte

Amos Winter

Maria Yang

Yong Zeng

Publisher's Note Springer Nature remains neutral with regard to jurisdictional claims in published maps and institutional affiliations. 